Modern Physics Letters A, Vol. 11, Nos. $39 \& 40$ (1996) 3127-3128

(C) World Scientific Publishing Company

\title{
ERRATA
}

\section{q-PHASE OPERATOR IN A FINITE-DIMENSIONAL HILBERT SPACE}

\author{
[Mod. Phys. Lett. A, Vol. 10, No. 4 (1995) 347-357]
}

Yaping Yang and Zurong Yu

On p. 348, Eqs. (12) and (13) are not correct for an $(s+1)$-dimensional space $V$ of the $q$-oscillator. The text in between Eq. (11) and Eq. (20) should be deleted. But Eq. (20) is correct. It can be derived from the following discussion.

Considering the eigenequation of the phase operator $\hat{\phi}$ in $V$,

$$
\hat{\phi}\left|\theta_{m}\right\rangle=\theta_{m}\left|\theta_{m}\right\rangle \quad \text { or } \quad \exp (i \hat{\phi})\left|\theta_{m}\right\rangle=\exp \left(i \theta_{m}\right)\left|\theta_{m}\right\rangle .
$$

Obviously, there exist $(s+1)$ independent eigenstates $\left\{\left|\theta_{m}\right\rangle, m=0,1,2, \ldots, s\right\}$ in $V$. Because the number state $\{|n\rangle, n=0,1,2, \ldots, s\}$ is a complete and orthonormal set in $V$, so we can write

$$
\left|\theta_{m}\right\rangle=\sum_{n=0}^{s} C_{n}^{(m)}|n\rangle, \quad m=0,1,2, \ldots, s .
$$

As $\left|\theta_{m}\right\rangle$ has to be complete and orthonormal

$$
\left\langle\theta_{m} \mid \theta_{m^{\prime}}\right\rangle=\delta_{m m^{\prime}}, \quad \sum_{m=0}^{s}\left|\theta_{m}\right\rangle\left\langle\theta_{m}\right|=1,
$$

we can obtain

$$
\sum_{n=0}^{s} C_{n}^{\left(m^{\prime}\right)} C_{n}^{(m) *}=\delta_{m m^{\prime}}
$$

and

$$
\sum_{n, n^{\prime}=0}^{s} \sum_{m=0}^{s} C_{n}^{(m) *} C_{n^{\prime}}^{(m)}\left|n^{\prime}\right\rangle\langle n|=1
$$


So we can reasonably assume that

$$
\sum_{m=0}^{s} C_{n}^{(m) *} C_{n^{\prime}}^{(m)}=\delta_{n n^{\prime}}
$$

From Eqs. (3a) and (4), we can choose coincidently

$$
\begin{aligned}
C_{n}^{(m)} & =\frac{1}{\sqrt{s+1}} \exp \left(i \theta_{m} n\right), \\
\theta_{m} & =\theta_{0}+\frac{2 m \pi}{s+1},
\end{aligned}
$$

where the value of $\theta_{0}$ is arbitrary. Using Eqs. (1), (5) and some mathematical formulas which are well known, we can easily obtained Eq. (20) and the other formulas after it.

Our paper gives an erroneous impression that "the Phase Quanta" concept had not been theoretically investigated before. However, the nondeformed phase quanta was proposed firstly and investigated in detail in Ref. 1.

\section{Reference}

1. V. Buzek et al., Phys. Rev. A45, 8079 (1992). 